\title{
Periódicos de bioética. ¿ڤHay muchos? ¿Hay pocos? ¿Cuál es la situación?
}

William Saad Hossne ${ }^{1}$

Resumen: Se intentó establecer un perfil bibliométrico de los periódicos de bioética en las bases de datos MEDLINE, LILACS, The Philosopher's Index, Ulrich's, SciELO y Qualis (Brasil). Los resultados destacados son: de 38 títulos indexados en MEDLINE, 25 (66\%) están en circulación; país con el número más grande: los EE.UU. (36\%); número más grande de periódicos de bioética apareció en la década de 1.990 (47\%), siendo que 36\% tienen menos de 10 años de existencia. Entre los periódicos extintos (34\%), el número medio de años es cerca de cuatro años. Solamente 12 países tienen periódicos indexados. En Brasil, de los tres periódicos de bioética sólo uno está indexado (artículos). El inglés es la lengua preponderante (45\%). Solamente 3\% de los periódicos son mensuales. En LILACS hay dos publicaciones más (Acta Bioethica, Chile, y Revista Latino Americana, Colombia). En la base SciELO, hay solamente Acta Bioethica. En la base Ulrich aparecen otras ocho publicaciones. En el "Qualis" hay solamente cuatro periódicos.

Palabras clave: periódicos científicos, publicaciones de bioética, perfil bibliométrico, bases de datos

\section{Bioethics journals: are there many? Are there few? What the situation is?}

\begin{abstract}
This work presents a bibliometric profile of Bioethics Journal according to the following journal databases: MEDLINE, LILACS, The Philosopher's Index, Ulrich's, SciELO and Qualis classification system (Brazil). We found that: from 38 titles indexed in MEDLINE, 25 (66\%) still exist; the country with the highest number is the U.S.A (36\%); most Bioethics journals appeared in the 1990 's (47\%), and 36\% have less than 10 years of existence. From those that disappeared (34\%), the average survival time was about 4 years. There are only 12 countries with indexed journals. In Brazil, from 3 Bioethics journals, only one is (partially) indexed. English is the preponderant language (45\%). Only 3\% of journals are monthly. LILACS has two additional publications (Acta Bioethica, Chile, and Revista Latino Americana, Colombia). SciELO has only Acta Bioethica. In Ulrich's database, there are eight additional publications. In "Qualis" classification system there are four journals.
\end{abstract}

Key words: scientific journal, Bioethics-publications, bibliometric profile, databases

\section{Periódicos de bioética. Muitos? Poucos? Como estamos?}

Resumo: Procurou-se traçar um perfil bibliométrico das revistas de Bioética a partir das bases: MEDLINE, LILACS, The Philosopher's Index, Ulrich's, SciELO e Qualis (Brasil). Ressaltam-se os seguintes resultados: de 38 títulos indexados no MEDLINE, 25 (66\%) estão em circulação; país com maior número E.U.A. (36\%); o maior número de periódicos de Bioética surgiu na década de 1.990 (47\%), sendo que 36\% têm menos de 10 anos de existência. Dos extintos (34\%), o tempo médio de sobrevida foi de cerca de 4 anos. São apenas 12 os países que têm periódicos indexados. No Brasil, das 3 revistas de Bioética, apenas uma é indexada (artigos). O inglês é o idioma preponderante (45\%). Apenas 3\% das revistas são mensais. Na base LILACS constam mais duas publicaçôes (Acta Bioethica, Chile, e Revista Latino Americana, Colômbia). Na base SciELO, apenas a Acta Bioethica. Na base Ulrich's figuram outras oito publicaçóes. Na "Qualis" constam somente 4 periódicos.

Palavras-chave: periódicos científicos, bioética-publicações, perfil bibliométrico, bases de dados

1 Profesor Emérito de la Escuela de Medicina de Botucatu (UNESP), Universidade Estadual Paulista, São Paulo, Brasil Correspondencia:secretariamestrado@saocamilo-sp.br 


\section{Introducción}

De neologismo aventado en 1927 por $\operatorname{Jahr}(1)$ y creado al principio de la década de 1970 por Potter(2), la bioética ha adquirido un cuerpo del doctrina y de actuación, aún en evolución franca, pero suficiente como área del conocimiento área esencialmente multidisciplinaria, pluralista que integra ciencias experimentales (sobre todo las biológicas) y las humanidades.

Su campo de actuación es bien amplio, incluyendo cuestiones éticas relativas a las ciencias de la vida, de la salud y del ambiente, sean cuestiones ya existentes (persistentes) o innovadoras (emergentes), fruto de los avances científicos. Por otra parte, es de la naturaleza de la bioética la preocupación ética por la humanidad, en el presente y el futuro.

En numerosos países y centros de investigación, y también de ayuda, se han creado asociaciones y/o sociedades de bioética, como también comisiones y/o comités de bioética. Congresos nacionales, regionales e internacionales han sido frecuentes, con el aumento del número de "bioeticistas". En diversos cursos de pre y de posgrado (en el área de la salud, sobre todo) se han incluido disciplinas de bioética. Se han publicado libros didácticos y de análisis de temas de bioética en todo el mundo.

Pero es principalmente, en los periódicos científicos, específicamente de bioética, que se espera encontrar consubstanciadas y debatidas reflexiones bioéticas actualizadas.

Parece válido intentar, desde ese contexto, evaluar el panorama relativo a esos periódicos, buscando establecer un cuadro descriptivo que conteste a las siguientes preguntas:

Cuál el número de periódicos específicamente de bioética (donde conste la palabra "bioética" en el título). ¿Son muchos? ¿Son pocos? ¿Están indexados en bases de datos? ¿Cuál la tasa de sobrevida de esos periódicos? ¿Cuál su regularidad? ¿En qué país se publican? ¿Cuáles se publican en Brasil?

Estas preguntas han constituido los objetivos de este artículo.

\section{Material y método}

\section{Bases de datos}

Para la investigación se han utilizado los siguientes términos: bioética, bioethica, bioéthique y bioetik en el título del periódico. La recopilación de datos fue hecha en las siguientes bases de datos:

\section{Medline}

La base de datos Medline se considera una de las más importantes de la literatura internacional en el área médica y biomédica. Engloba más de 7.000 títulos de periódicos publicados en EE.UU. y en otros 70 países.

Para la indexación de un periódico en esa base de datos se consideran factores como propósito, calidad del contenido, calidad del trabajo editorial y calidad de la producción publicada(3).

Contiene cerca de 11 millones de registros de literatura desde 1966(4); de América Latina están indexados en general 53 periódicos, siendo $18 \mathrm{del}$ Brasil. Se considera relativamente baja la representatividad de América Latina(5).

\section{Lilacs}

Literatura Latinoamericana y del Caribe y en Ciencias de la Salud fue creada en 1985, en sustitución al Index Medicus Latino Americano (IMLA), creado en 1978. Engloba 37 países de América Latina y del Caribe. Registra artículos de periódicos, monografías académicas, libros y capítulos de libros relacionados con el área de la salud. Indexa cerca de 1.000 títulos.

\section{Philosopher's Index}

En el área de la filosofía, es una excelente base de datos. Incluye 15 campos de la filosofía, incluso periódicos y libros publicados desde 1940; se indexan 570 periódicos, de 43 países.

\section{Ulrich's Periodical Directory}

La publicación se inició en 1932 como "Periodical Directory”. Incluye más que 300.000 periódicos de todas las áreas; abarca todos los temas, 
así como publicaciones regulares o irregulares. Es una base de datos empresarial y comercial.

\section{SciELO (Scientific Eletronic Library Online)}

El modelo de SciELO, creado el 1997, resultó de la cooperación entre la Fundação de Amparo à Pesquisa do Estado de São Paulo (FAPESP) y la Bireme, Centro Latino Americano e do Caribe de Informação em Ciências da Saúde. Hoy el modelo incluye 205 periódicos (disponibles en http:// www.scielo.org).

\section{Sistema "Qualis" - CAPES}

Se cotejaran los datos, particularmente los obtenidos en la base de datos de Medline, con la clasificación Qualis de la Coordinación de Perfeccionamiento del Personal de Nivel Superior (CAPES - versión 2009). El sistema Qualis es un sistema brasileño considerado para la evaluación bibliométrica en cursos de posgrado.

Según Silva(6), los primeros estudios acerca de la evaluación de periódicos en el Brasil aparecieron en la década de 1960. El sistema "Qualis" ha sido revisado recientemente (2009, disponible en http://www.capes.gov.br). Clasifica los periódicos (nacionales e internacionales) en las categorías A1, A2, B1 - B5 y C, atribuyendo diversos pesos (en orden decreciente) a cada una de las categorías. Un mismo periódico puede recibir conceptos diferentes de acuerdo con el comité del área del conocimiento donde si insertan los programas de posgrado (maestría y doctorado).

\section{Variables analizadas}

Usando las palabras clave, se intentó conseguir en estas bases de datos la siguiente información:

- Título completo del periódico (hoy).

- Fecha de creación.

- Alteración del nombre - fecha.

- Fecha del cierre (cuando es el caso).

- Periodicidad.

- Lengua.

- País de edición.

En los casos de cambio de nombre, se consideró como fecha de creación la del nombre actual del periódico. El estudio analítico se centró en los datos del sistema Medline; en las otras bases de datos, el estudio tuvo naturaleza descriptiva y complementaria.

\section{Resultados}

\section{Medline}

\section{Número de periódicos}

Se catalogaran 38 periódicos en cuyo título aparece la palabra "bioética". La cifra incluye más de una vez un periódico cuando se ha modificado el título respectivo, así como cuando el periódico ya no es publicado. De entre los 38 periódicos, sólo 25 (66\%) están en circulación (activa) y indexados en MEDLINE (PUBMED).

Para fines de comparación, se presenta el número de periódicos indexados en Medline en algunas áreas. Cirugía: 349, psiquiatría: 155, neurología: 115, pediatría: 97, ginecología: 93, ética: 93, dermatología: 23 y oftalmología: 70.

De los 25 periódicos de bioética, siete no están indexados en su totalidad, sino solamente artículos seleccionados.

\section{Fecha de creación}

En la década de 1970 aparecieron dos periódicos (ambos en 1976); en la década del 80, nueve periódicos; en la década de 1990, 18 periódicos, y en la década de 2000, nueve periódicos.

Desde 1976 (fecha de la creación del primer periódico) hasta 2008 (siendo el más reciente publicado en 2005) se presentan en Medline, como se ha dicho, 38 periódicos; en circulación en este momento hay 25 , es decir, cerca de un tercio de los periódicos (34\%) ya no se publican o han cambiado de nombre; el 36\% tiene menos de diez años de existencia.

3. Sobrevida media de los periódicos desaparecidos

En su totalidad, el tiempo de sobrevida (hasta la extinción o cambio del nombre) es 3,8 años; el período menor es un año y el mayor 13 ańos. 
4. País nativo de los periódicos (tabla 1)

Tabla 1 - País nativo de los periódicos (incluyendo los extintos)

\begin{tabular}{|l|r|r|}
\hline \multicolumn{1}{|c|}{ País } & Número & \multicolumn{1}{c|}{$\%$} \\
\hline Estados Unidos & 17 & $45 \%$ \\
\hline Inglaterra & 4 & $11 \%$ \\
\hline Australia & 3 & $8 \%$ \\
\hline Nueva Zelandia & 3 & $8 \%$ \\
\hline España & 2 & $5 \%$ \\
\hline Canadá & 2 & $5 \%$ \\
\hline Colombia & 1 & $3 \%$ \\
\hline Rumania & 1 & $3 \%$ \\
\hline Francia & 1 & $3 \%$ \\
\hline Eslovaquia & 1 & $3 \%$ \\
\hline Holanda & 1 & $3 \%$ \\
\hline Bélgica & 1 & $3 \%$ \\
\hline Brasil & 1 & $3 \%$ \\
\hline
\end{tabular}

De los 13 periódicos desaparecidos (o con alteración de nombre), ocho (el 61\%) son de Estados Unidos, dos de Canadá, uno de Inglaterra, uno de Australia y uno de Nueva Zelandia.

5. Periódicos aún en circulación registrados en Medline

\subsection{Número y distribución por país.}

Según lo ya examinado, hoy circulan 25 periódicos con la palabra bioética en el título.

Los periódicos según el país nativo se distribuyen de la siguiente manera: Estados Unidos nueve (36\%); Australia tres (12\%); Inglaterra, Nueva Zelandia y España dos (8\%), Francia, Eslovaquia, Bélgica, Holanda, Rumania, Colombia y Brasil uno (4\%).

Solamente 12 países del mundo publican periódicos de bioética indexados en Medline.

Los periódicos más antiguos con el nombre actual se publican en Estados Unidos desde 1985 (Ethics and Medicine, A Christian perspective on issues in Bioethics) y en Inglaterra desde 1987 (Bioethics).

Los dos más recientes surgieron en 2005, ambos en Estados Unidos (Annals of Bioethics y Penn Bioethics Journal).

\subsection{En cuanto a la periodicidad}

No fue posible establecer con precisión la periodicidad de todos los 25 periódicos en circulación; se nota irregularidad en algunos.
Se analizó la periodicidad de 21 de ellos: 29\% son trimestrales, 29\% cuatrimestrales, 29\% semestrales y $3 \%$ mensuales.

\subsection{En cuanto al factor de impacto}

El factor de impacto más elevado es el de American J. Bioethics (3.934), seguido por Bioethics (1.52). Hay que recordar que el más elevado globalmente es el de Nature $(28,751)$ y Science $(26,372)$.

\subsection{En cuanto a la lengua}

Predomina el uso del idioma inglés (45\%), siguiendo el español y el francés (5\%); otros idiomas son el portugués, el eslovaco y el rumano.

\section{Lilacs}

Están registrados seis periódicos, en los cuales la expresión "bioética" aparece en el título. Dos de éstos están en MEDLINE: Bioética (Brasil, Consejo Federal de Medicina) y Persona y Bioética (España).

De los otros cuatro, dos están indexados: Revista Latino Americana de Bioética (Bogotá-Colombia) y Acta Bioethica (Chile, CIEB, iniciado en 2000).

\section{Philosopher's Index}

En esa base de datos aparecen tres periódicos indexados con la expresión "bioética" en el título; los tres también están en Medline (Bioethics, Bioethics Fórum, Bioethics Quarterly).

\section{ULRICH'S}

Se mencionan 39 títulos, 31 de ellos están en Medline. Los otros ocho son:

1. The Ag Bioethics Fórum

2. Bioethics Outlook

3. Bibliography of Bioethics

4. Bioethics Literature Review

5. Bioethics Thesaurus

6. Canadian Bioethics Report

7. Global bioethics

8. Int. J. of Feminist Approaches to Bioethics 


\section{SciELO}

En esta base hay solamente un título: Acta Bioethica (Chile-CIEB).

\section{Evaluación de CAPES - clasificación}

De los 25 periódicos de PUBMED, solo cuatro están en la evaluación de CAPES, con los índices respectivos:

1. Bioethics (Inglaterra): B.1 (Comité Interdisciplinario)

2. Developing World Bioethics (Inglaterra): B3 (Comité Interdisciplinario), B5 (Comité de salud pública).

3. Eubios J. of Asian and Int. Bioethics (Nueva Zelandia): C (Comité de Biotecnología), B4 (Comité Interdisciplinario), B5 (Comité de salud colectiva).

4. Bioética e Ética Médica (Brasil): B3 (Comité Interdisciplinario, Comité Salud Social), B4 (Comité Odontología), B5 (Comité filosofía I), B6 (Comité Medicina II), C (Comité Derecho), C (Comité Salud colectiva).

En la clasificación de CAPES se incluyen siete periódicos no indexados en Medline y cuatro de ellos tampoco están en las bases examinadas aquí (Bioética Seleciones, Cadernos de Bioética, Perspectivas Bioéticas, Cuadernos de Bioética).

Los siete periódicos son:

1. Revista Brasileira de Bioética (Brasil): B4 (Comité Engenharia), B4 (Comité Interdisciplinario), B6 (Comité Ed. Física), B6 (Comité Enfermagem), B5 (Comité Filosofía), B5 (Comité Medicina I), B6 (Comité Medicina II), C (Comité Derecho), C (Comité Salud Colectiva)

2. Acta Bioethica (Chile): B3 (Comité Odontología)

3. Revista Bioethikos (Brasil): B4 (Comité Interdisciplinario), B5 (Comité Educación) y B5 (Comité Enfermería)

4. Bioética Seleciones: B4 (Comité S. Colectiva)

5. Cadernos de Bioética (Brasil): B5 (Comité Interdisciplinario)
6. Perspectivas Bioéticas: C (Comité Medicina II) y C (Comité S. Social)

7. Cuadernos de Bioética (Argentina): B5 (Comité Derecho)

\section{Consideraciones finales}

En su condición de nueva área del conocimiento, la bioética viene construyendo un cuerpo conceptual y doctrinal aún en franca evolución. En poco más de 30 años existen ya contribuciones académico-científicas sólidas que proceden de bioeticistas del mundo entero. Es creciente el número de sociedades, congresos, comisiones o comités, además de la producción científica en el área de bioética. Por lo tanto, parece oportuno lanzar una mirada a los medios (periódicos) de difusión de la bioética.

La bioética ha ocupado su propio territorio, ha crecido y se ha desarrollado al punto de merecer, aunque de manera incipiente, una evaluación bibliométrica dentro del campo amplio de la cientometría. Esta última palabra probablemente ha surgido, al igual que la palabra "bioética", como neologismo; de hecho, como señala Vanti(7), la cientometía debe de haber surgido en la antigua URSS, propuesta por Dobrov y Karennoi en 1969; ha ganado mayor identidad en la década de 1970, con la publicación del periódico Scienciometrics, publicado original en Hungría y hoy en Holanda(7). Como rama de la sociología de la ciencia(8) ha originado otros neologismos, como informetría, webmetría y bibliometría.

Según Saes(9), el trabajo de los indicadores bibliométricos permite determinar aspectos relevantes en la evaluación de la producción científica, como por ejemplo el crecimiento de campos de la ciencia, la productividad de autores, instituciones y países.

Es con esa preocupación, con el objetivo básico de evaluar el perfil de los periódicos en el área de la bioética, que quisimos desarrollar este artículo. De ahí la opción por las bases y los indicadores bibliométricos. Utilizando las palabras "bioética" y "bibliometria" no se han encontrado referencias bibliográficas en Medline. 
Un primer resultado que merece consideración se vincula con el número de periódicos específicos de bioética que han sido creados y el número de los periódicos "extintos". Han emergido 38 periódicos y 25 siguen existiendo (Medline). Por otra parte, considerando la amplia difusión de las actividades bioéticas en el mundo, el número cada vez mayor de bioeticistas, sociedades y comités de bioética, así como la amplia penetración de la bioética en programas de investigación y enseñanza (sobre todo en el área biomédica), el número de periódicos parece inferior a las expectativas.

Hay un hecho que sobresale: ya en el 1976 (después de cinco años de existencia de la palabra "bioética"), surgen dos periódicos, ambos en EE.UU., uno nacido con el nombre Bioethics Northwest, "muerto" en 1977 debido a cambios de nombre, retornando el 1988 con el nombre J. Medical Humanities, y otro llamado Bioethics Digest que desapareció en 1978.

Destacable es el número relativamente elevado de periódicos creados en la década del 90 (18) equivalente a la suma del número de periódicos creados en las décadas del 80 y del 2000. Hay que seńalar, igualmente, que de los 38 periódicos indexados en Medline, 13 (34\%) se han extinguido o cambiado de nombre, sobreviviendo sólo 25; el tiempo medio antes de la "extinción" es cerca de cuatro años. El índice más grande de "extinción" ocurrió en la década del 80 (67\%).

Otro hecho resaltable es que 25 periódicos indexados en Medline son publicados por sólo 12 países, la mayoría por EE.UU. (36\%), así como la ausencia de periódicos indexados en esta base y publicados en países como Canadá, Italia, Alemania, Portugal, Suecia, Rusia, Japón y otros.

También se percibe la "pobreza relativa" de periódicos de América Latina y el Caribe, y en las otras bases, como Lilacs y SciElo. El que destaca es Acta Bioethica (Chile).

En cuanto a la situación brasileña, se ha verificado que de los 25 periódicos en circulación indexados en Medline, hay sólo un periódico (Revista Bioética e Ética Médica), publicado desde 1993 (con el nombre actual, desde 2005); por otra parte, la indexación es sólo de algunos artículos, no del pe- riódico como un todo.

En Brasil se publican dos otros periódicos: Revista Brasileira de Bioética (Sociedad Brasileña de Bioética) desde 2005, y Bioethikos (Centro Universitario Sáo Camilo) desde 2007. Ambos con equipos altamente calificados y con alta calidad de contenido.

En 1992, se creó Cadernos de Bioética (PUC, Minas Gerais), extinguido el 2000. Se conocen varios otros, publicados por diversos países que, no obstante, no han tenido (o no han merecido) las condiciones para la indexación en esas bases de datos. Algunos han publicado artículos de gran interés para la escena nacional e internacional.

De acuerdo con el progreso en bioética en cada país o región, puede más ser indicada y oportuna la publicación de artículos en periódicos dirigidos al público adecuado, a pesar de que el periódico no esté indexado en Medline. Lo mismo puede ocurrir en función del tema de la publicación y a quién ella se destina, para proponer debates entre el público o para la divulgación y consolidación de núcleos de bioética.

Obviamente, los periódicos deben presentar condiciones adecuadas de calidad y términos de contenido, producción y publicación. Sin embargo, los periódicos todavía no indexados en las bases de datos principales merecen respecto y ayuda, según sus propias calidades, y deben ser apoyados para alcanzar desarrollo. No nos parece adecuado, principalmente desde una perspectiva ética, ignorar esos periódicos. Por el contrario, los que presentan potencialidades de desarrollo deben merecer la atención debida.

Sin duda, no se deben hacer concesiones en el campo científico; se debe exigir calidad, así como mejoría permanente. Por otra parte, hay que evaluar y, cuando sea indicado, practicar una apertura para evitar un círculo vicioso paralizante y obstaculizador de la evolución y mejoría, como señaló bien Mueller(10) hace ya 10 años.

\section{Agradecimientos}

A las bibliotecarias Aparecida Regina Denadai Silva, Enilze de Souza Nogueira Volpato y Nivaldete Costa Fernandes Cruz. 


\section{Referencias}

1. Jahr F. Bioéthik Eline Unschou uber die ethischen Beziehungen des Menschen zu Tier und Pflanze. Kosmo 1997;24:2-4.

2. Potter VR. Bioethics: bridge to the future. EUA: Prentice Hall; 1971.

3. Martinez JAB. Perseguindo o MEDLINE. J Bras Pneumol 2005; 31: 95-96.

4. Pizzani L. O estado da arte da produção cientifica em educação especial na Biblioteca Virtual em Saúde (BVS): um estudo bibliométrico (dissertação). São Carlos (SP): Universidade Federal de São Carlos; 2008.

5. Pellizon RF, Poblacón DA, Goldenberg S. Pesquisa na área da saúde: seleção das principais fontes para acesso à literatura científica. Acta Cir Bras 2003; 18: 493-496.

6. Silva RC. Indicadores bibliométricos da produção científica em educação especial: Estudo da Revista "Educação Especial" (2000-2006) (dissertação). São Carlos (SP): Universidade Federal de São Carlos; 2008.

7. Vanti NAP. Da bibliometria à webmetria: uma exploração conceitual dos mecanismos utilizados para medir o registro da informática e a difusão do conhecimento. Revista Ciência da Informação 2002; 3: 152-162.

8. Macias Chapula CA. O papel da informetria e da cientometria e sua perspectiva nacional e internacional. Revista Ciência da Informaçâo 1998; 27: 134-140.

9. Saes SG. Estudo bibliométrico das publicaçôes em Economia da saúde no Brasil-1989/1998. Dissertação de Mestrado. São Paulo: Faculdade de Saúde Pública, Universidade de São Paulo; 2000.

10. Mueller SPM. O círculo vicioso que prende os periódicos nacionais. Revista Ciência da Informação 1999. Disponível em: http://www.dgz.org.br

Recibido: 26 de septiembre de 2009

Aceptado: 13 de enero de 2010 